\title{
Functional tricuspid regurgitation at the time of mitral valve repair for degenerative leaflet prolapse: The case for a selective approach
}

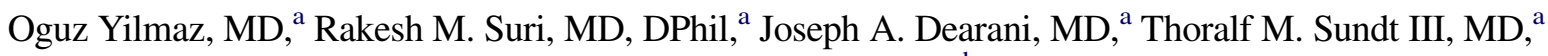 \\ Richard C. Daly, MD, ${ }^{\mathrm{a}}$ Harold M. Burkhart, MD, ${ }^{\mathrm{a}}$ Zhuo Li, MS, ${ }^{\mathrm{b}}$ Maurice Enriquez-Sarano, MD, ${ }^{\mathrm{c}}$ and \\ Hartzell V. Schaff, MD
}

\begin{abstract}
Objectives: It is not clear whether clinically silent tricuspid valve regurgitation should be addressed at the time of mitral valve repair for severe mitral regurgitation due to leaflet prolapse. We examined the clinical and echocardiographic outcomes of patients with tricuspid regurgitation who underwent only mitral valve repair.
\end{abstract}

\begin{abstract}
Methods: We retrospectively analyzed records of patients undergoing mitral valve repair for isolated mitral valve prolapse who had coexistent tricuspid valve regurgitation during an 11-year period at our institution. Echocardiographic data were compared preoperatively, intraoperatively, and postoperatively at less than 1,1 to 3,3 to 5 , and more than 5 years.

Results: In 699 patients who underwent mitral valve repair for severe mitral regurgitation, mean age was 60.4 years and $459(66 \%)$ were male. At the time of mitral valve repair, tricuspid valve regurgitation was grade 3 or more in $115(16 \%)$ patients and less than grade 3 in $584(84 \%)$ patients. After mitral valve repair, overall grade of tricuspid valve regurgitation decreased significantly within the first year $(P=.01)$. In patients with grade 3 regurgitation or more, the grade decreased at dismissal and until the third year $(P<.001)$. Female sex, preoperative atrial fibrillation, and diabetes mellitus were independent risk factors for increased tricuspid valve regurgitation with time; preoperative regurgitation of grade 3 or more independently predicted decreased grade with time. Only 1 patient required tricuspid reoperation 4.5 years after mitral repair.
\end{abstract}

Conclusions: Clinically silent nonsevere tricuspid valve regurgitation in patients with degenerative mitral valve disease is unlikely to progress after mitral valve repair. Tricuspid valve surgery is rarely necessary for most patients undergoing repair of isolated mitral valve prolapse. (J Thorac Cardiovasc Surg 2011;142:608-13)

Functional tricuspid valve regurgitation (TR) is common in patients with left-sided cardiac valve disease. ${ }^{1,2}$ Although early reports suggested that TR may resolve after the diseased mitral valve (MV) is replaced, ${ }^{1}$ subsequent data have shown that severe TR may develop late after MV surgery for rheumatic or ischemic disease, even in the absence of significant residual mitral stenosis, regurgitation, or other causes of left-sided heart failure. ${ }^{2-4}$ Severe symptomatic residual TR compromises long-term outcomes after MV surgery and is associated with increased early and midterm morbidity and mortality, despite adequate MV correction. ${ }^{2-6}$ However, previous studies have focused mainly on patients with ischemic, rheumatic, or mixed heart valve disease undergoing MV replacement, ${ }^{7-10}$ and their conclusions

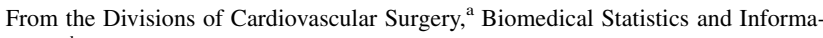
tics, ${ }^{\mathrm{b}}$ and Cardiovascular Diseases, ${ }^{\mathrm{c}}$ Mayo Clinic, Rochester, Minn. Disclosures: Authors have nothing to disclose with regard to commercial support. Oguz Yilmaz and Rakesh M. Suri have contributed equally to the manuscript. Received for publication Jan 20, 2010; revisions received Sept 16, 2010; accepted for publication Oct 24, 2010; available ahead of print Jan 31, 2011.

Address for reprints: Rakesh M. Suri, MD, DPhil, Division of Cardiovascular Surgery, Mayo Clinic, 200 First St SW, Rochester, MN 55905 (E-mail: suri. rakesh@mayo.edu).

$0022-5223 / \$ 36.00$

Copyright (C) 2011 by The American Association for Thoracic Surgery doi:10.1016/j.jtcvs.2010.10.042
}

cannot be readily extended to patients undergoing isolated repair for degenerative MV disease. Specifically, few data have addressed the progression of clinically silent, functional TR and the need for reoperation after repair of mitral leaflet prolapse. It is therefore unclear whether the tricuspid valve (TV) should be addressed at the time of isolated MV repair. We analyzed the clinical and echocardiographic outcomes of patients with clinically silent functional TR in whom only MV repair was performed.

\section{PATIENTS AND METHODS}

We searched our prospective patient database for the records of patients who underwent MV repair for isolated MV prolapse and who had coexistent, clinically silent, functional TR between January 1, 1995, and January 1, 2006, at Mayo Clinic, Rochester, Minnesota. The study was reviewed and approved by the Mayo Clinic Institutional Review Board. The need for individual patient consent was waived because relevant identifiers were not included in the dataset. We excluded patients who declined involvement in clinical research, who had concomitant coronary artery bypass grafting surgery, or who had other concomitant cardiac procedures other than closure of a patent foramen ovale. Also excluded were patients with an initial diagnosis of MV regurgitation (MR) caused by congenital, rheumatic, or ischemic heart disease or cardiomyopathy and those with endocarditis causing leaflet defects or subvalvular abscess. Patients with primary pulmonary disease, significant right ventricular dysfunction, or structural TV abnormalities (including stenosis) were also not included. Fifty-two patients who had TR associated with right heart failure 


$$
\begin{aligned}
& \text { Abbreviations and Acronyms } \\
& \text { MR }=\text { mitral valve regurgitation } \\
& \text { MV }=\text { mitral valve } \\
& \text { SD }=\text { standard deviation } \\
& \text { TR }=\text { tricuspid valve regurgitation } \\
& \text { TV }=\text { tricuspid valve }
\end{aligned}
$$

symptoms and/or TV leaflet disease underwent TV surgery over the same interval, and these patients were analyzed in a separate report.

All patients underwent MV repair for severe MR owing to leaflet prolapse. The techniques used during MV repair have been described elsewhere. ${ }^{11}$ The most common method of surgical correction for posterior leaflet prolapse was triangular resection and suture reconstruction of the involved scallop, supplemented by a standard-length $(63-\mathrm{mm})$ flexible posterior annuloplasty band. For repair of anterior leaflet prolapse, artificial polytetrafluoroethylene neochordae were used. Chordal shortening, chordal transfer, commissural annuloplasty, and leaflet plication were used infrequently.

TR in each patient was quantified echocardiographically as follows: grade 1, trivial; grade 2, mild; grade 3, moderate; and grade 4, severe. TR data were analyzed during the following time periods: preoperatively, intraoperatively, and postoperatively at less than 1 year, 1 to 3 years, 3 to 5 years, and more than 5 years. Clinical follow-up data were collected through routine postoperative surveys (at 1, 3, 5, 10, 15, and 20 years) and contact with referring cardiologists.

\section{STATISTICAL ANALYSIS}

Descriptive statistics for categorical variables are reported as frequency and percentage, and continuous variables are given as mean (standard deviation [SD]). The Kaplan-Meier method was used to estimate 5-year and 10 -year survival. Changes in TR from preoperative values to those at dismissal and postoperatively at less than 1 year, 1 to 3 years, 3 to 5 years, and more than 5 years of follow-up were analyzed on the basis of the subgroup of patients whose data were available at the corresponding time periods. Changes were compared using paired $t$ tests or the Wilcoxon rank sum test as appropriate. Predictors of echocardiographic change in TR after 5 years of follow-up were identified by fitting linear regression models. The multivariate model considered univariately significant variables $(P<.05)$ with model selection using the stepwise method (backward and forward techniques were similar). Cox regression models were used to determine univariate and multivariate predictors of late mortality. All statistical tests were 2 -sided, with the $\alpha$ level set at .05 for statistical significance.

\section{RESULTS}

A total of 699 patients undergoing isolated MV repair met the inclusion criteria for the study. The mean (SD) age was 60.4 (13.7) years, and $459(65.7 \%)$ were male. Preoperatively, most patients $(457,65.4 \%$ ) had New York Heart Association class I-II symptoms, whereas 242
(34.6\%) had class III-IV symptoms. The baseline characteristics of the patients are shown in Table 1. MV leaflet prolapse was posterior in 377 patients $(53.9 \%)$, whereas $280(40.1 \%)$ had bileaflet prolapse and $42(6 \%)$ had isolated anterior leaflet prolapse. No patients had right heart failure, and although all patients had some degree of coexistent functional TR, none was deemed by the surgeon to require TV intervention at the time of MV repair. According to preoperative echocardiography, $115(16 \%)$ patients had grade 3 or higher TR, and the remaining $584(84 \%)$ had less than grade 3 TR.

\section{Intraoperative TR Evaluation}

In the 115 patients with grade 3 or higher TR preoperatively, we compared TR grade at different time points related to surgery: preoperative, intraoperative prebypass, intraoperative postbypass, and predismissal (Figure 1). Mean intraoperative TR grades while patients were under general anesthesia (both prebypass and postbypass) were significantly lower than both preoperative and predismissal levels $(P<.001$ for both $)$.

\section{Change in TR During Follow-up}

The mean (SD) duration of follow-up for the group was 5.5 (3) years. TR grade was compared at different time periods after surgery (Figure 2, $A$ ). The mean preoperative TR grade was $1.84(0.71)$. Compared with the preoperative value, overall mean TR grade decreased significantly within the first year $(\mathrm{n}=248 ; 1.72[0.79] ; P=.01)$ and increased slightly only after 5 years of follow-up $(\mathrm{n}=108 ; 2.11$ [0.92]; $P=.03$ ). There was no significant change from preoperative levels at the other time periods examined.

TABLE 1. Patient baseline characteristics

\begin{tabular}{lc}
\hline \multicolumn{1}{c}{ Characteristic } & Value* $(\mathbf{N}=\mathbf{6 9 9})$ \\
\hline Age, y & $60.4(13.7)$ \\
Male sex & $459(65.7)$ \\
Preoperative EF, \% & $65.12(7.57)$ \\
Preoperative TR grade & \\
1 & $233(33.3)$ \\
2 & $351(50.2)$ \\
$3-4$ & $115(16.5)$ \\
Preoperative AF & $122(17.5)$ \\
Preoperative dilatation & \\
RA & $203(29.0)$ \\
RV & $31(4.4)$ \\
NYHA class & \\
I & $199(28.5)$ \\
II & $258(36.9)$ \\
III & $220(31.5)$ \\
IV & $22(3.1)$ \\
\hline
\end{tabular}

$E F$, Ejection fraction; $T R$, tricuspid valve regurgitation; $A F$, atrial fibrillation; $R A$, right atrium; $R V$, right ventricle; $N Y H A$, New York Heart Association. *Values are no. (\%) or mean (standard deviation). 


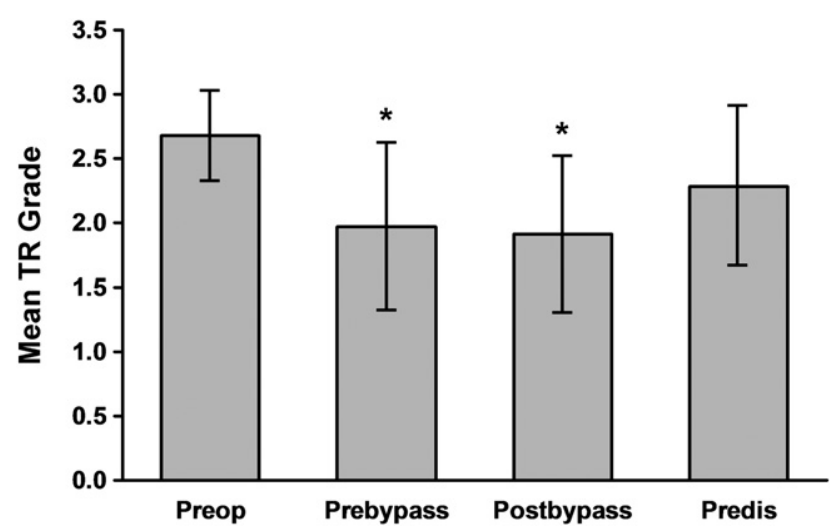

FIGURE 1. Mean grade of tricuspid valve regurgitation $(T R)$ at different points relative to surgery for patients with preoperative grade 3-4 TR $(\mathrm{n}=115)$. Preop, Preoperative; Predis, predismissal. $* P<.001$ (paired $t$ test or Wilcoxon rank sum test).

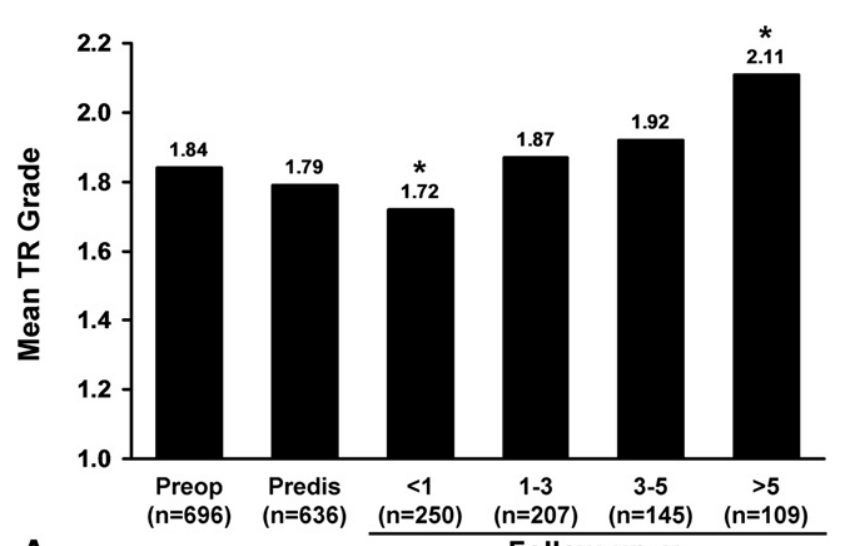

A

Follow-up, y

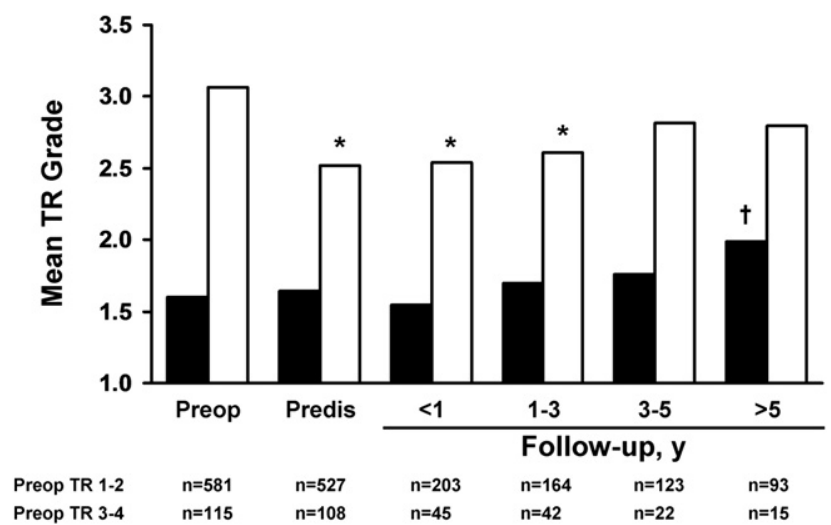

\section{B}

FIGURE 2. Mean grade of tricuspid valve regurgitation $(T R)$ at different time points. A, All patients. B, All patients separated by preoperative grade of TR: grade 1-2, black bars; grade 3-4, white bars. Preop, Preoperative; Predis, predismissal. $* P<.001 ; \dagger P<.01$ (paired $t$ test or Wilcoxon rank sum test).
Change in TR from preoperative values was then reassessed after stratification of the study population into 2 groups: those with preoperative TR less than moderate $(\mathrm{n}=581)$ and those with moderate or greater TR $(\mathrm{n}=115)$ (Figure $2, B)$. In patients with less than moderate preoperative TR (mean grade, 1.6 [0.49]), mean TR grade remained stable and increased only slightly after 5 years (mean, $2.0[0.86] ; P<.01$ ). Conversely, in those with at least moderate preoperative TR, mean TR grade decreased significantly from preoperative values after MV repair $(P<.001$ at dismissal, $<1$ year, and $1-3$ years $)$. Mean TR grade was also decreased after 3 years of follow-up, but the changes were not statistically significant $(P=.18$ after 3 years; $P=.33$ after 5 years). Only 1 reoperation (TV repair) for severe symptomatic TR was necessary 4.5 years after the initial MV operation. The patient had moderate preoperative TR at the time of MV repair, and significant right ventricular dysfunction developed shortly after surgery. The distributions of functional TR and recurrent MR with time are shown in Appendix Table 1.

\section{Predictors of Change in TR}

Univariate analysis was performed and multivariate models were constructed to determine the influence of preoperative and intraoperative variables on change in TR after MV repair (Table 2$)$. Female sex $(P=.009)$, preoperative atrial fibrillation $(P<.001)$, and comorbid diabetes mellitus

TABLE 2. Predictors of change in TR grade*

\begin{tabular}{lccccc}
\hline & \multicolumn{2}{c}{ Univariate } & & \multicolumn{2}{c}{ Multivariate } \\
\cline { 2 - 3 } \cline { 5 - 6 } \multicolumn{1}{c}{ Parameter } & \multicolumn{3}{c}{ Parameter } & & $\begin{array}{c}\text { Parameter } \\
\text { estimate }\end{array}$ \\
\hline Female sex & .02 & 0.405 & .009 & 0.441 \\
Age & .37 & 0.006 & & \\
Hypertension & .08 & 0.312 & & \\
Diabetes mellitus & .02 & 1.181 & .02 & 1.112 \\
Previous AF & $<.001$ & 0.642 & $<.001$ & 0.628 \\
Preoperative echocardiography & & & & \\
RA dilatation & .03 & 0.577 & & \\
RV dilatation & .18 & 0.510 & & \\
EF & .10 & -0.017 & & \\
LVMI & .67 & -0.001 & & \\
LAVI & .56 & 0.005 & & \\
PAP & .71 & 0.003 & & \\
$\quad$ TR velocity & .64 & 0.100 & & \\
Preoperative TR (grade 3-4) & .03 & -0.525 & .008 & -0.614 \\
Operative finding, MV prolapse & & & & \\
Anterior & .03 & -1.200 & & \\
Posterior & .03 & -0.893 & & \\
Both & .07 & -0.762 & & \\
\hline
\end{tabular}

$T R$, Tricuspid valve regurgitation; $A F$, atrial fibrillation; $R A$, right atrium; $R V$, right ventricle; $E F$, ejection fraction; $L V M I$, left ventricular mass index; $L A V I$, left atrial volume index; PAP, peak pulmonary arterial pressure; $M V$, mitral valve. ${ }^{*}$ Change in TR grade from preoperative values to those after $>5$ years of follow-up. 
$(P=.02)$ were all independent predictors of increased TR more than 5 years after MV repair. The presence of at least moderate TR preoperatively was associated with a decrease in TR with time $(P=.008)$. Pulmonary arterial pressure was not predictive of a change in TR during follow-up.

\section{Predictors of Mortality}

There were 4 early deaths $(0.6 \%), 2(0.3 \%)$ of which were within 30 days of surgery. Survivals at 5 and 10 years after surgery were $93.9 \%$ and $76.1 \%$, respectively. Multivariate analysis identified only older age (hazard ratio, $2.63 ; P<.001)$ as a predictor of decreased long-term survival (Table 3). The degree of preoperative TR was not associated with early or late mortality.

\section{DISCUSSION}

We evaluated the clinical and echocardiographic outcomes of a homogeneous population of patients with MV prolapse and clinically silent functional TR undergoing isolated MV repair and found that clinically important TR progression is unlikely to occur after repair of the MV in this specific category of patients. Female sex, diabetes mellitus, and a preoperative history of atrial fibrillation were all independently associated with slight echocardiographic progression of TR over time; however, this was clinically insignificant and did not lead to excess risk of reoperation. Our data suggest that, in the absence of right heart failure, surgical TV intervention for functional TR is rarely necessary in patients undergoing isolated MV repair for degenerative leaflet prolapse.

TABLE 3. Univariate and multivariate predictors of late mortality

\begin{tabular}{lccccc}
\hline \multirow{2}{*}{ Variable } & \multicolumn{2}{c}{ Univariate } & & \multicolumn{2}{c}{ Multivariate } \\
\cline { 2 - 3 } Female sex & HR & $\boldsymbol{P}$ value & & HR & $\boldsymbol{P}$ value \\
Age & 1.09 & $<.001$ & & 2.63 & $<.001$ \\
Hypertension & & .37 & & \\
Diabetes mellitus & & .19 & & \\
Renal failure & & .54 & & \\
COPD & & .007 & & \\
Previous AF & & .22 & & \\
Preoperative TR $\geq$ grade 3 & & .02 & & \\
RA dilatation & & .24 & & \\
RV dilatation & & .28 & & \\
EF & 0.98 & .19 & & \\
LVEDD & 0.93 & .005 & & \\
LVESD & 0.97 & .33 & & \\
LAVI & 1.01 & .20 & & \\
LVMI & 1.01 & .23 & & \\
\hline
\end{tabular}

$H R$, Hazard ratio; $C O P D$, chronic obstructive pulmonary disease; $A F$, atrial fibrillation; $T R$, tricuspid valve regurgitation; $R A$, right atrium; $R V$, right ventricle; $E F$, ejection fraction; $L V E D D$, left ventricular end-diastolic dimension; $L V E S D$, left ventricular end-systolic dimension; $L A V I$, left atrial volume index; $L V M I$, left ventricular mass index.
MV repair is the standard of care in patients with degenerative MV disease. ${ }^{12}$ Patients who undergo MV repair have normalization of life expectancy, have long-term freedom from reoperation equivalent to that with mechanical valve replacement, and avoid the need for valve-related anticoagulation therapy. ${ }^{12}$ Recent data suggest that even in patients with recurrent MR owing to primary repair failure, MV rerepair is more efficacious than valve replacement in promoting long-term regression of left ventricular dimensions and improvement in patient survival. ${ }^{12}$

Coexistent TR is common in patients referred for surgery for MV disease. ${ }^{13,14}$ Factors that have been shown to increase the likelihood of TR progression include left ventricular dysfunction, right ventricular dilatation or dysfunction, pulmonary hypertension, and atrial fibrillation. ${ }^{15-17}$ Although early reports suggested that functional TR might resolve spontaneously after MV replacement, ${ }^{1}$ it was subsequently discovered that some patients may have relentless progression of TR leading to right heart failure necessitating reoperation. This possibility is important because recurrent severe TR has been shown to influence the long-term outcome of patients treated for left heart pathologic processes, ${ }^{15,18}$ and reoperation in this setting may be associated with high early and late mortality. ${ }^{2-4,9}$ These findings have led to a liberal approach to TV repair in patients with TR who are undergoing MV surgery. ${ }^{2,4}$ Indeed, some clinicians have recommended repair of coexistent TR on the basis of echocardiographic severity ${ }^{8}$ or tricuspid annular dimension. ${ }^{19}$

An important question is whether functional TR progresses after correction of all types of left-sided valve disease. The difficulty with generalizing a response and creating a unified approach to functional TR is that the underlying MV disease processes are heterogeneous. Mechanisms of left-sided valve disease are disparate and range from rheumatic ${ }^{7}$ to ischemic and other cardiomyopathic processes. ${ }^{10}$ Few data address coexistent TR in those with the most frequent MV condition in the Western world-degenerative MV leaflet prolapse. It is for this reason that the current study was performed.

Our data demonstrate that the progression of TR in patients with isolated MV leaflet prolapse is unusual; the degree of TR decreased within the first year overall and increased only slightly after 5 years (Figure 2, A). Even at 5 years, however, the mean TR grade remained mild and, as most would likely agree, clinically insignificant. It also was surprising that TR grade for those in whom TR might have been expected to increase (patients with at least moderate preoperative TR) actually decreased significantly during the first 3 years and remained lower than the preoperative level overall (Figure 2, B). In patients with less than moderate TR, however, TR was relatively stable over time. Only 1 patient in our series had development of severe symptomatic TR necessitating reoperation more 
than 4 years after initial MV repair. In contrast, in patients with rheumatic or ischemic MV disease, clinically significant late TR has been shown to develop in $37 \%$ to $49 \%,{ }^{3,10}$ and we agree with an aggressive approach to TV repair in these patients.

Three factors were found to be independently associated with an increase in TR with time in our series: female sex $(P=.009)$, preoperative atrial fibrillation $(P<.001)$, and comorbid diabetes mellitus $(P=.02)$ (Table 2). Previous reports have identified atrial fibrillation, a large left atrium, severe preoperative $\mathrm{TR},{ }^{8}$ older age, ${ }^{3,4}$ and female $\operatorname{sex}^{3}$ as important predictors of late TR after left-sided cardiac surgery. Although neither right nor left atrial size was associated with increased TR in the current analysis, atrial fibrillation was predictive. In a recent study by Kwak and associates ${ }^{17}$ examining the outcomes of 615 patients undergoing MV or MV and aortic valve surgery, preoperative atrial fibrillation was also identified as the only important predictor of late TR. Their study had important differences from ours, such as their inclusion of patients with both stenotic and mixed MV lesions as well as those undergoing aortic valve replacement. That study also found that patients with severe TR had decreased long-term survival, ${ }^{17}$ which differs from our own finding that survival is similarly high regardless of follow-up TR grade. We suggest that the discrepancies between these 2 reports are largely related to differences in the populations analyzed. Finally, it is interesting that pulmonary artery pressure was not an independent predictor of TR progression in our series. It is widely understood, however, that the etiology of functional secondary TR is multifactorial and that some with pulmonary hypertension may never have development of significant TR. ${ }^{20}$

We found that moderate preoperative TR was a predictor of decreased TR over time $(P=.008)$ (Table 2$)$ after correction of MR. This also differs from previously studied populations with varied MV pathologic processes, in which TR higher than grade 2 was a risk factor for long-term TR progression. ${ }^{3,17}$ Whereas others have suggested that altered right ventricular geometry and TV annular dilatation are causative factors in the progression of late TR after MV surgery for cardiomyopathy-related disease, ${ }^{21-23}$ it is tempting to speculate that those with isolated MV leaflet prolapse undergoing early valve repair may be less prone to such changes because of the minimization of structural remodeling of the interventricular myocardium. Given that atrial fibrillation influences TR progression, the concept of a myopathic influence on tricuspid annular size and function is intriguing. Our group is currently testing this hypothesis.

The study in the literature most similar to ours, published by Dreyfus and associates ${ }^{19}$ in 2005 , evaluated 311 patients undergoing MV repair: 163 (52.4\%) underwent MV repair alone, and 148 (47.6\%) additionally had TV repair because of a tricuspid annular diameter of $70 \mathrm{~mm}$ or greater. They found no difference between these groups in late survival, cardiac-related events, or reoperation. However, TR progressed more frequently in those without TV intervention, and New York Heart Association class was slightly worse in this group. Several important differences exist between that study and our own. First, whereas $100 \%$ of the patients in our study had isolated MR owing to degenerative leaflet prolapse, $35 \%$ of those in the study reported by Dreyfus and associates ${ }^{19}$ had different MV disease related to ischemia, rheumatic disease, endocarditis, or other cardiomyopathy. It is widely understood that the natural history of these diseases can be significantly discrepant; for example, there is evidence to support progressive generalized valvulopathy in rheumatic disease. In contrast, it is unclear why tricuspid annular dilation in association with degenerative MV prolapse, which alone is incapable of causing significant TR, at the time of MV repair would cause subsequent development of significant TR during follow-up. Finally, New York Heart Association functional class is a poor measure of right-sided heart failure symptoms related to significant uncorrected functional TR.

It should again be noted that the current study examines a homogeneous group of patients with severe degenerative MR and some degree of coexistent TR to determine how the TV in those with left-sided heart disease owing to MV leaflet prolapse might behave differently from that in patients with mixed MV disease presented in prior reports. A recent review by Rogers and Bolling ${ }^{24}$ reaffirms that the incidence of TR after MV repair is dependent on the cause of MR. Whereas Matsuyama and colleagues ${ }^{8}$ demonstrated that only $16 \%$ of those undergoing MV repair for nonischemic disease had moderate-severe TR at follow-up, Matsunaga and Duran ${ }^{10}$ reported swift progression to greater than moderate TR in $74 \%$ at more than 3 years of follow-up. It is clear that the cause of left-sided heart disease influences the incidence and progression of TR. Surgical decisionmaking in the current era should reflect this important fact.

\section{LIMITATIONS}

This study is a retrospective review of prospectively collected data and, as such, is influenced by typical biases. It would have been ideal to compare the results of a similar group of patients with isolated MV leaflet prolapse who underwent concomitant TV intervention, but the small number of such patients in our clinical experience $(n=52)$ precludes a scientifically rigorous comparison. Although patients with MV prolapse who have a second related valve disease (eg, significant secondary TR) are typically referred for repeat echocardiography and clinical/surgical reassessment at our institution, we acknowledge the possibility that some within this population who had symptomatic recurrent TR may have sought care elsewhere and were thus undetected by our routine follow-up. Furthermore, although 
we were not able to specifically determine the incidence of right heart failure symptoms, our practice in general is to offer surgery for symptomatic functional TR. Because of the length of time over which this study was completed, there are variations in the frequency and extent of echocardiographic follow-up. Finally, the technical ability to reproducibly quantify TR also improved over the course of the study as echocardiographic technology evolved.

\section{CONCLUSIONS}

Asymptomatic nonsevere functional TR found in association with severe MR caused by MV leaflet prolapse may not require surgical correction at the time of $\mathrm{MV}$ repair. Although the cost of TV intervention may at times be low, a cogent evidence-based surgical strategy is warranted in this group of patients. A thoughtful approach to the TV should consider preoperative risk factors such as atrial fibrillation, sex, and diabetes mellitus. Further study is necessary to understand the complex relationship between left- and rightsided heart disease in this important patient population.

\section{References}

1. Braunwald NS, Ross J Jr, Morrow AG. Conservative management of tricuspid regurgitation in patients undergoing mitral valve replacement. Circulation. 1967;35(4 Suppl):I63-9.

2. King RM, Schaff HV, Danielson GK, Gersh BJ, Orszulak TA, Piehler JM, et al. Surgery for tricuspid regurgitation late after mitral valve replacement. Circulation. 1984;70(3 Pt 2):I193-7.

3. Porter A, Shapira Y, Wurzel M, Sulkes J, Vaturi M, Adler Y, et al. Tricuspid regurgitation late after mitral valve replacement: clinical and echocardiographic evaluation. J Heart Valve Dis. 1999;8:57-62.

4. Staab ME, Nishimura RA, Dearani JA. Isolated tricuspid valve surgery for severe tricuspid regurgitation following prior left heart valve surgery: analysis of outcome in 34 patients. J Heart Valve Dis. 1999;8:567-74.

5. Breyer RH, McClenathan JH, Michaelis LL, McIntosh CL, Morrow AG. Tricuspid regurgitation: a comparison of nonoperative management, tricuspid annuloplasty, and tricuspid valve replacement. J Thorac Cardiovasc Surg. 1976;72: 867-74.

6. Groves PH, Lewis NP, Ikram S, Maire R, Hall RJ. Reduced exercise capacity in patients with tricuspid regurgitation after successful mitral valve replacement for rheumatic mitral valve disease. Br Heart J. 1991;66:295-301.

7. Izumi C, Iga K, Konishi T. Progression of isolated tricuspid regurgitation late after mitral valve surgery for rheumatic mitral valve disease. J Heart Valve Dis. 2002; 11:353-6.
8. Matsuyama K, Matsumoto M, Sugita T, Nishizawa J, Tokuda Y, Matsuo T. Predictors of residual tricuspid regurgitation after mitral valve surgery. Ann Thorac Surg. 2003;75:1826-8.

9. Cohen SR, Sell JE, McIntosh CL, Clark RE. Tricuspid regurgitation in patients with acquired, chronic, pure mitral regurgitation, II: nonoperative management, tricuspid valve annuloplasty, and tricuspid valve replacement. J Thorac Cardiovasc Surg. 1987;94:488-97.

10. Matsunaga A, Duran CM. Progression of tricuspid regurgitation after repaired functional ischemic mitral regurgitation. Circulation. 2005;112(9 Suppl): I453-7.

11. Seccombe JF, Schaff HV. Mitral valve repair: current techniques and indications In: Franco KL, Verrier ED, eds. Advanced therapy in cardiac surgery. Hamilton (ON): BC Decker; 1999:220-31.

12. Suri RM, Schaff HV, Dearani JA, Sundt TM 3rd, Daly RC, Mullany CJ, et al. Recurrent mitral regurgitation after repair: should the mitral valve be re-repaired? J Thorac Cardiovasc Surg. 2006;132:1390-7. Epub 2006 Nov 20.

13. Colombo T, Russo C, Ciliberto GR, Lanfranconi M, Bruschi G, Agati S, et al. Tricuspid regurgitation secondary to mitral valve disease: tricuspid annulus function as guide to tricuspid valve repair. Cardiovasc Surg. 2001;9:369-77.

14. Cohn LH. Tricuspid regurgitation secondary to mitral valve disease: when and how to repair. J Card Surg. 1994;9(2 Suppl):237-41.

15. Nath J, Foster E, Heidenreich PA. Impact of tricuspid regurgitation on long-term survival. J Am Coll Cardiol. 2004;43:405-9.

16. Calafiore AM, Gallina S, Iaco AL, Contini M, Bivona A, Gagliardi M, et al. Mitral valve surgery for functional mitral regurgitation: should moderate-or-more tricuspid regurgitation be treated? A propensity score analysis. Ann Thorac Surg. 2009;87:698-703.

17. Kwak JJ, Kim YJ, Kim MK, Kim HK, Park JS, Kim KH, et al. Development of tricuspid regurgitation late after left-sided valve surgery: a single-center experience with long-term echocardiographic examinations. Am Heart J. 2008;155: 732-7. Epub 2008 Feb 21.

18. De Bonis M, Lapenna E, Sorrentino F, La Canna G, Grimaldi A, Maisano F, et al Evolution of tricuspid regurgitation after mitral valve repair for functional mitral regurgitation in dilated cardiomyopathy. Eur J Cardiothorac Surg. 2008;33: 600-6. Epub 2008 Feb 12.

19. Dreyfus GD, Corbi PJ, Chan KM, Bahrami T. Secondary tricuspid regurgitation or dilatation: which should be the criteria for surgical repair? Ann Thorac Surg. 2005;79:127-32.

20. Mutlak D, Aronson D, Lessick J, Reisner SA, Dabbah S, Agmon Y. Functional tricuspid regurgitation in patients with pulmonary hypertension: is pulmonary artery pressure the only determinant of regurgitation severity? Chest. 2009;135: 115-21. Epub 2008 Aug 21.

21. Groves PH, Ikram S, Ingold U, Hall RJ. Tricuspid regurgitation following mitral valve replacement: an echocardiographic study. J Heart Valve Dis. 1993;2: 273-8.

22. Kim HK, Kim YJ, Park JS, Kim KH, Kim KB, Ahn H, et al. Determinants of the severity of functional tricuspid regurgitation. Am J Cardiol. 2006;98:236-42. Epub 2006 May 30.

23. Fukuda S, Gillinov AM, Song JM, Daimon M, Kongsaerepong V, Thomas JD, et al. Echocardiographic insights into atrial and ventricular mechanisms of functional tricuspid regurgitation. Am Heart J. 2006;152:1208-14.

24. Rogers JH, Bolling SF. The tricuspid valve: current perspective and evolving management of tricuspid regurgitation. Circulation. 2009;119:2718-25.

APPENDIX TABLE 1. Distribution of functional TR and residual MR over time

\begin{tabular}{|c|c|c|c|c|c|}
\hline \multirow[b]{2}{*}{ Follow-up time } & \multicolumn{3}{|c|}{ TR } & \multicolumn{2}{|c|}{ MR } \\
\hline & Total & Grade $<\mathbf{3}^{*}$ & Grade $\geq 3 *$ & Total & Grade $\geq 3^{*}$ \\
\hline Preoperative & 696 & $581(83.5)$ & $115(16.5)$ & 699 & $699(100)$ \\
\hline Predismissal & 627 & $517(82.4)$ & $110(17.5)$ & 679 & $26(3.8)$ \\
\hline$\leq 1 \mathrm{y}$ & 250 & $212(84.8)$ & $38(15.2)$ & 278 & $16(5.8)$ \\
\hline $1-3 y$ & 207 & $165(79.7)$ & $42(20.3)$ & 219 & $18(8.2)$ \\
\hline $3-5 y$ & 145 & $112(77.2)$ & $33(22.8)$ & 151 & $12(7.9)$ \\
\hline$>5 y$ & 109 & 77 (70.6) & $32(29.4)$ & 110 & $11(10)$ \\
\hline
\end{tabular}

$M R$, Mitral valve regurgitation; $T R$, tricuspid valve regurgitation. *Values are no. of patients $(\%)$. 